\title{
GMR
}

\section{Differentiation between Triatoma arthurneivai and Triatoma wygodzinskyi (Hemiptera: Reduviidae: Triatominae) using cytotaxonomy}

\author{
K.C.C. Alevi ${ }^{1}$, C.H.L. Imperador ${ }^{1}$, F.F.F. Moreira ${ }^{2}$, J. Jurberg ${ }^{2}$ and \\ M.T.V. Azeredo-Oliveira ${ }^{1}$ \\ ${ }^{1}$ Laboratório de Biologia Celular, Instituto de Biociências, \\ Letras e Ciências Exatas, \\ Universidade Estadual Paulista "Júlio de Mesquita Filho", \\ São José do Rio Preto, SP, Brasil \\ ${ }^{2}$ Laboratório Nacional e Internacional de Referência em Taxonomia de \\ Triatomíneos, Instituto Oswaldo Cruz, Rio de Janeiro, RJ, Brasil \\ Corresponding author: K.C.C. Alevi \\ E-mail: kaiochaboli@hotmail.com
}

Genet. Mol. Res. 15 (2): gmr.15027869

Received October 21, 2015

Accepted December 22, 2015

Published June 21, 2016

DOI http://dx.doi.org/10.4238/gmr.15027869

\begin{abstract}
Using classic morphometric techniques to examine the head and thorax of Triatoma specimens, researchers identified a possible taxonomic problem involving T. arthurneivai (Lent \& Martins) and $T$. wygodzinskyi (Lent). A recent geometric morphometric study indicated that the insects captured outside the Serra do Cipó region, State of Minas Gerais, Brazil, were T. wygodzinskyi. The misidentification of $T$. arthurneivai as $T$. wygodzinskyi could result in several problems associated with entoepidemiological lifting, the biological characterization of the species, and phylogenetic reconstruction. For the first time, we describe the use of cytogenetic analysis as a tool for differentiation between T. arthurneivai and T. wygodzinskyi. The results indicated that both species had the same number of chromosomes $2 \mathrm{n}$ $=22(20 \mathrm{~A}+\mathrm{XY})$. However, analyses of spermatocytes during early
\end{abstract}


prophase indicated that it was possible to differentiate T. arthurneivai and T. wygodzinskyi, because only T. arthurneivai exhibited heteropycnotic blocks distributed in the chromatin. Therefore, we highlight the analysis of spermatocytes as a taxonomic tool for the characterization of $T$. arthurneivai and $T$. wygodzinskyi, and suggest that the technique can be used for entoepidemiological lifting in vector control programs. Thus, the results presented here, in conjunction with morphometric analyses, are of utmost taxonomic and epidemiological importance for the identification of T. arthurneivai and T. wygodzinskyi specimens.

Key words: Cytogenetics; Taxonomy; Maculata subcomplex

\section{INTRODUCTION}

The description of Triatoma arthurneivai (Lent \& Martins) was based on a single female specimen collected in Serra do Cipó, State of Minas Gerais, Brazil (Lent and Martins, 1940). After approximately 10 years, Pellegrino (1951) collected specimens of this species in Santa Rita de Caldas, in southern Minas Gerais. Using the specimens collected by Pellegrino (1951), Lent (1951) described T. wygodzinskyi (Lent). In addition to those collected in Minas Gerais, specimens first identified as T. arthurneivai were also collected in the States of São Paulo [Sorocaba (Corrêa et al., 1962) and Itupararanga/Votorantim (Forattini et al., 1968)] and Paraná (Stumpf et al., 1981).

Using classic morphometric techniques to examine the head and thorax, Dos Santos et al. (2007) suggested a possible taxonomic problem involving T. arthurneivai and $T$. wygodzinskyi. Indeed, a recent geometric morphometric study showed that the insects captured outside the Serra do Cipó region were T. wygodzinskyi specimens (Carbajal de la Fuente et al., 2011). These authors reported that the populations of T. arthurneivai from São Paulo, which were studied for more than 40 years by several authors (Corrêa et al., 1962, 1965; Pinto Alves and Noda, 1964; Juarez, 1970; Forattini et al., 1968, 1972; Barretto and Ribeiro, 1981; Hypsa et al., 2002; de Paula et al., 2005; Rosa et al., 2005; Dos Santos et al., 2007; Bargues et al., 2008), were actually T. wygodzinskyi specimens.

The misidentification of T. arthurneivai as T. wygodzinskyi could result in several problems associated with entoepidemiological lifting (Pinto Alves and Noda, 1964), the biological characterization of the species (Forattini et al., 1968; Juarez, 1970), and phylogenetic reconstruction (Hypsa et al., 2002; de Paula et al., 2005; Bargues et al., 2008). The identification of new inexpensive tools that aid the correct identification of the species is of great epidemiological importance. Therefore, we describe the use of cytogenetic analysis as a tool for differentiation of T. arthurneivai and T. wygodzinskyi.

\section{MATERIAL AND METHODS}

At least two adult males from each species (T. arthurneivai and T. wygodzinskyi) were used, and the specimens were assigned by the insectariums of the National and International Laboratory of Reference for Triatominae Taxonomy, Oswaldo Cruz Institute (FIOCRUZ), Rio de Janeiro, Brazil (T. arthurneivai) and the Laboratory of Triatomines and Chagas Disease Epidemiology at the René Rachou Research Center (CPqRR/FICRUZ), Minas Gerais, Brazil (T. wygodzinskyi). 
The biological material used to characterize cells was spermatocytes, which were easily obtained from testicular material by tearing the seminiferous tubules of adult males prior to fixation to a cover slip. The samples were then subjected to a cytogenetic technique that utilized lacto-acetic orcein (De Vaio et al., 1985, with modifications based on Alevi et al., 2012). At least 50 cells from each species were analyzed using a Jenaval light microscope (Zeiss) attached to a digital camera and an Axio Vision LE 4.8 image analyzer (Copyright 2006-2009 Carl Zeiss Imaging Solutions Gmb H), and the obtained images were magnified by a factor of $1000 \mathrm{X}$.

\section{RESULTS}

The results of the analyses indicated that both species had the same number of chromosomes $2 \mathrm{n}=22(20 \mathrm{~A}+\mathrm{XY})$. However, analyses of spermatocytes during early prophase indicated that it was possible to differentiate T. arthurneivai and T. wygodzinskyi, because several heteropycnotic blocks distributed in the chromatin were present in T. arthurneivai (Figure 1A) but not in T. wygodzinskyi (Figure 1B). In addition, both species have a chromocenter formed by the $\mathrm{X}$ and $\mathrm{Y}$ sex chromosomes (Figure 1A and B, arrows).

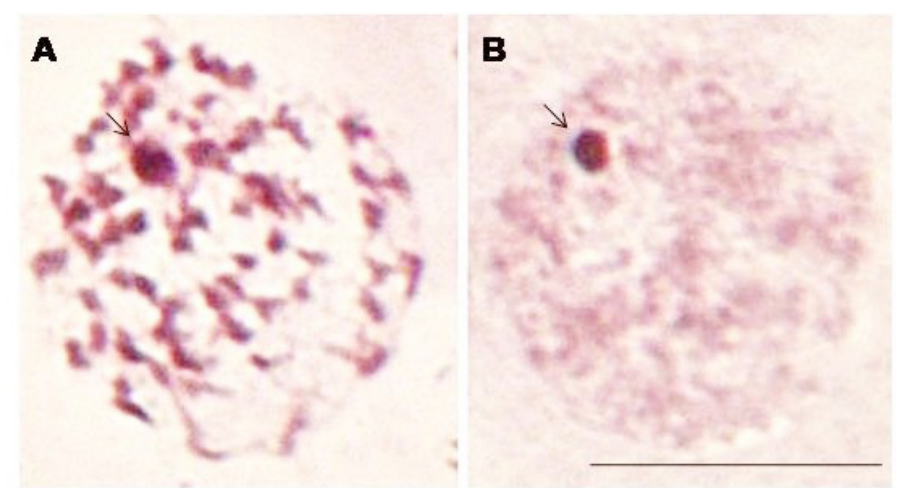

Figure 1. Early prophase of Triatoma arthurneivai (A) and T. wygodzinskyi (B). Note that T. arthurneivai exhibits several heteropycnotic blocks distributed in the chromatin, but these are not present in T. wygodzinskyi. Arrow: chromocenter formed by the X and Y sex chromosomes. Scale bar: $10 \mu \mathrm{m}$.

\section{DISCUSSION}

All species belonging to the Maculata subcomplex (T. maculata, T. pseudomaculata, T. arthurneivai, and T. wygodzinskyi) have the same number of chromosomes $(2 \mathrm{n}=22 ; 20 \mathrm{~A}$ $+\mathrm{XY}$ ) (Dos Santos et al., 2007). Dos Santos et al. (2007) also examined the meiotic prophase stages of T. arthurneivai, T. maculata, and T. pseudomaculata, and the authors found that the T. arthurneivai spermatocyte exhibited a heteropycnotic chromocenter formed by the sex chromosomes. However, our results confirmed that the insects analyzed by Dos Santos et al. (2007) were T. wygodzinskyi specimens, which corroborated the conclusion proposed by Carbajal de la Fuente et al. (2010).

The cytogenetic characterization of triatomine spermatocytes was also important for the differentiation between T. melanocephala and other members of the Brasiliensis 
subcomplex (Alevi et al., 2013, 2014), between T. maculata and T. pseudomaculata (Dos Santos et al., 2007), between T. guasayana and T. sordida (Panzera et al., 1997), and between T. rubrofasciata and other 30 species of the triatomines (Alevi et al., 2016). The cellular analyses of spermatocytes allowed the characterization of many species-specific patterns, thereby ensuring that morphologically related species were differentiated, so as observed for T. arthurneivai and T. wygodzinskyi.

The cytogenetic technique that utilized lacto-acetic orcein was cheaper and faster than molecular methods. This colorant has an affinity for basic structures, so histone and nonhistone proteins involved in compaction of the genetic material (heteropycnotic blocks) are stained, and this characteristic was of great importance for the cytogenetic and cytotaxonomic studies of triatomines (Ueshima, 1966; De Vaio et al., 1985). The analysis of spermatocytes using orcein as a taxonomic tool for the characterization of T. arthurneivai and T. wygodzinskyi specimens was highlighted here, and we suggest that it may be used for entoepidemiological lifting in vector control programs.

Therefore, the combination of cytotaxonomic and morphometric analyses (Carbajal de la Fuente et al., 2011) were proven to be of utmost taxonomic and epidemiological importance for the identification of T. arthurneivai and T. wygodzinskyi specimens.

\section{Conflicts of interest}

The authors declare no conflict of interest.

\section{ACKNOWLEDGMENTS}

Research supported by Fundação de Amparo à Pesquisa do Estado de São Paulo (FAPESP; Process \#2013/19764-0) and Conselho Nacional de Desenvolvimento Científico e Tecnológico (CNPq).

\section{REFERENCES}

Alevi KCC, Mendonça PP, Pereira NP, Rosa JA, et al. (2012). Karyotype of Triatoma melanocephala Neiva and Pinto (1923). Does this species fit in the Brasiliensis subcomplex? Infect. Genet. Evol. 12: 1652-1653. http://dx.doi. org/10.1016/j.meegid.2012.06.011

Alevi KCC, Mendonça PP, Pereira NP, Rosa JA, et al. (2013). Spermatogenesis in Triatoma melanocephala (Hemiptera: Triatominae). Genet. Mol. Res. 12: 4944-4947. http://dx.doi.org/10.4238/2013.October.24.5

Alevi KCC, Rosa JA and Azeredo-Oliveira MTV (2014). Cytotaxonomy of the Brasiliensis subcomplex and the Triatoma brasiliensis complex (Hemiptera: Reduviidae: Triatominae). Zootaxa 3838: 583-589. http://dx.doi.org/10.11646/ zootaxa.3838.5.7

Alevi KCC, Nascimento JGO, Moreira FFF, Jurberg J, et al. (2016). Cytogenetic characterization of Triatoma rubrofasciata (De Geer) (Hemiptera, Triatominae) spermatocytes and its cytotaxonomic application. Afr. Entomol., in press.

Bargues MD, Klisiowicz DR, Gonzalez-Candelas F, Ramsey JM, et al. (2008). Phylogeography and genetic variation of Triatoma dimidiata, the main Chagas disease vector in Central America, and its position within the genus Triatoma. PLoS Negl. Trop. Dis. 2: e233. http://dx.doi.org/10.1371/journal.pntd.0000233

Barretto MP and Ribeiro RD (1981). Estudo sobre reservatórios o vetores silvestres do Trypanosoma cruzi. LXXVII. Observações sobre a ecologia do Triatoma arthurneivai Lent \& Martins, 1940 (Hemiptera, Reduviidae). Rev. Bras. Biol. 41: 317-320.

Carbajal de la Fuente AL, Jaramillo N, Barata JMS, Noireau F, et al. (2011). Misidentification of two Brazilian triatomes, Triatoma arthurneivai and Triatoma wygodzinskyi, revealed by geometric morphometrics. Med. Vet. Entomol. 25: 178-183. http://dx.doi.org/10.1111/j.1365-2915.2010.00912.x 
Corrêa RR, Alves AP and Pascale G (1962). Presença do Triatoma arthurneivai em Sorocaba (Hemiptera, Reduviidae). Rev. Paul. Med. 60: 267.

Corrêa RR, Pinto Alves U and Noda J (1965). Nota sobre o Triatoma arthurneivai. Seu criadouro extradomiciliar (Hemiptera, Reduviidae). Rev. Brasil. Malar. 17: 217-234.

de Paula AS, Diotaiuti L and Schofield CJ (2005). Testing the sister-group relationship of the Rhodniini and Triatomini (Insecta: Hemiptera: Reduviidae: Triatominae). Mol. Phylogenet. Evol. 35: 712-718. http://dx.doi.org/10.1016/j. ympev.2005.03.003

De Vaio ES, Grucci B, Castagnino AM, Franca ME, et al. (1985). Meiotic differences between three triatomine species (Hemiptera, Reduviidae). Genetica 67: 185-191. http://dx.doi.org/10.1007/BF02424489

Dos Santos SM, Lopes CM, Dujardin JP, Panzera F, et al. (2007). Evolutionary relationships based on genetic and phenetic characters between Triatoma maculata, Triatoma pseudomaculata and morphologically related species (Reduviidae: Triatominae). Infect. Genet. Evol. 7: 469-475. http://dx.doi.org/10.1016/j.meegid.2007.01.008

Forattini OP, Juarez E and Rabello EX (1968). Dados sôbre a biologia do Triatoma arthurneivai no sudeste do estado de São Paulo, Brasil (Hemiptera, Reduviidae). Rev. Saude Publica 2: 186-193. http://dx.doi.org/10.1590/S0034$\underline{89101968000200005}$

Forattini OP, Rabello EX and Pattoli DB (1972). Aspectos ecológicos da tripanossomose americana. IV. Mobilidade de Triatoma arthurneivai em seus ecótopos naturais. Rev. Saude Publica 6: 183-187. http://dx.doi.org/10.1590/S0034$\underline{89101972000200008}$

Hypsa V, Tietz DF, Zrzavý J, Rego RO, et al. (2002). Phylogeny and biogeography of Triatominae (Hemiptera: Reduviidae): molecular evidence of a New World origin of the Asiatic clade. Mol. Phylogenet. Evol. 23: 447-457. http://dx.doi. org/10.1016/S1055-7903(02)00023-4

Juarez E (1970). Observações sôbre o ciclo evolutivo do Triatoma arthurneivai, em condições de laboratório (hemiptera, Reduviidae). Rev. Saude Publica 4: 13-18. http://dx.doi.org/10.1590/S0034-89101970000100003

Lent H (1951). Novo Triatoma no Estado de Minas Gerais (Brasil). (Hemiptera: Reduviidae). Rev. Bras. Entomol. 22: 349-353.

Lent H and Martins V (1940). Estudos sobre os triatomíneos do Estado de Minas Gerais, com descrição de uma nova espécie. Rev. Entomol 2: 877-886.

Panzera F, Hornos S, Pereira J, Cestau R, et al. (1997). Genetic variability and geographic differentiation among three species of Triatomine bugs (Hemiptera-Reduviidae). Am. J. Trop. Med. Hyg. 57: 732-739.

Pellegrino J (1951). Transmissores da doença de Chagas no estado de Minas Gerais. Rev. Assoc. Med. Minas Gerais 2: $43-66$.

Pinto Alves U and Noda J (1964). Os transmissores da doença de Chagas da região de Sorocaba no Estado de São Paulo, Brasil. Arq. Hig. 29: 141-157.

Rosa JA, Medeiros MP, Cilense M and Barata JMS (2005). Morphological study of the thorax of the five nymphal instars of Triatoma arthurneivai Lent \& Martins (Hemiptera, Reduviidae, Triatominae). Rev. Bras. Entomol. 49: 289-293. http://dx.doi.org/10.1590/S0085-56262005000300001

Stumpf IVK, Luz E and Borba AM (1981). Triatoma arthurneivai e Rhodnius neglectus no Estado do Paraná. (Redviidae, Triatominae). Arq. Biol. Tecnol. 24: 301-303.

Ueshima N (1966). Cytotaxonomy of the triatominae (Reduviidae: Hemiptera). Chromosoma 18: 97-122. http://dx.doi. org/10.1007/BF00326447 